\title{
5. Fazit und Ausblick
}

»The meaning and significance of an amateur video are found in how the community responds to it«, schreibt Strangelove (2010: 47) in Bezug auf die Videopraxis auf YouTube. Hieran anschließend lässt sich im diffraktiven Blick dieser Arbeit formulieren: Die Bedeutung und Bedeutsamkeit eines Handy-Todesvideos und eines davon ausgehenden Diffraktionsmusters zeigt sich darin, welche Narrative sich mit ihm aufspannen und sich über ästhetische und diskursive Figurationen mit anderen verbinden. Ästhetik und Diskurs sind im narrativen Netz der Handy-Todesvideos eng versponnen. In diesem Netz bilden die Figurationen an der Schnittstelle Tod-Handyvideo-Syrienkonflikt zwar feste, aber keineswegs unbewegliche Knoten. An diesen laufen einzelne Narrative innerhalb des Phänomens der Handy-Todesvideos zusammen, verflechten sich zu einem Netz von Erzählungen und Figurationen und bringen eine figurativ-narrative Praxis der Wissensund Bedeutungsgenerierung hervor. Gleichzeitig bilden die Erzählungen die Verbindungen zwischen den Figurationen, die diese mal eng und mal weniger eng verflechten. In jedem Fall bilden sie ein dichtes Gewebe des figurativ-narrativen Netzes der Handy-Todesvideos, das wir im ersten folgenden Abschnitt entlang der sich darin entfaltenden Politik der Bedeutung abschließend betrachten. Im zweiten Abschnitt begehen wir noch einmal die diffraktive Perspektive, die unseren Blick in dieser Arbeit geleitet hat. Wir schauen insbesondere auf die Schnittstellen und Affinitäten, die auf das Außerhalb dieser Perspektive verweisen und diese Arbeit anschlussfähig für weitere wissenschaftliche Betrachtungen machen.

\subsection{Die Ästhetisierung und Politisierung des Todes: Eine Politik der Bedeutung}

In unserem diffraktiven Blick auf das Phänomen der Handy-Todesvideos zeigt sich, dass das Filmen, Veröffentlichen und Betrachten des eigenen Todes und des Todes des Anderen in eine Politik der Bedeutung eingebunden sind, in der eine Ästhetisierung und Politisierung des Todes untrennbar ineinandergreifen. Diese Arbeit positioniert sich dabei nicht innerhalb des Syrienkonflikts als einen international 
geführten politischen Konflikt, sondern stellt die Handy-Todesvideos als medienund kulturwissenschaftlich relevantes Phänomen in den Mittelpunkt. So lautet die zentrale Fragestellung auch gerade nicht Was bedeutet der Tod und das Handyvideo im Syrienkonflikt?, sondern: Was bedeutet der Tod, das Handyvideo und der Syrienkonflikt im Handy-Todesvideos? Abseits einer Positionsbeziehung zu und in einem internationalgeführten politischen Konflikt ist diese Arbeit jedoch insofern politisch, als dass sie sich gegenüber einer Politik der Bedeutung im Sinn von regulierenden ästhetischen und diskursiven Regimen der Bedeutungserzeugung, zuschreibung und -auslöschung der Handy-Todesvideos positioniert. Das Phänomen der Handy-Todesvideos ist dabei nicht nur innerhalb des Syrienkonflikts situiert. Über komplexe figurative Verflechtungen und vielfältige ästhetische und diskursive Verschränkungen weist es über diesen hinaus: bis zurück zu dem Foto des erschossenen Benno Ohnesorg von 1967, der im Rahmen der westdeutschen Studentenbewegung zur Ikone wurde, und bis hin zu rechtspopulistischen Strömungen, die in Antwort auf die europäische Flüchtlingskrise ab 2015 mit ihrer aggressiven Identitätspolitik immer stärker in den Raum des Sicht- und Sagbaren eingetreten sind.

Hierbei werden nicht nur ganz unterschiedliche, sondern auch gegengelagerte und umschlagende Motivationen und Wahrnehmungen hervorgebracht. Ästhetische und diskursive Widerständigkeiten verbinden sich mit Affekten und Authentifizierungen, mit Verwerfungen und Falsifizierungen, mit Parodie und Fake. In der Politik der Bedeutung der Handy-Todesvideos ist der Tod zugleich lebensbedeutend und lebensbedrohend, unterhaltend und schockierend, abstoßend und faszinierend, betrauerbar und verspottbar. Das Handyvideo ist zugleich dokumentarisch und pornografisch, bezeugend und fabrizierend, anklagend und kriminell, enthüllend und exponierend. Der Syrienkonflikt ist zugleich Überlebenskampf und Identitätskampf, Erlebnis und Horror, Realität und Spiel. Wie in einem Kippbild kann dabei die eine Wahrnehmung nicht ohne die andere gesehen werden, wenn sie einmal wahrgenommen wurde. Der Ästhetisierung und Politisierung des Todes in den Handy-Todesvideos wohnt damit der Effekt einer nachträglichen Unwiderruflichkeit inne. In der Frühphase des Konflikts konnten die >First-person<Handy-Todesvideos noch fast ausschließlich als eine politische Waffe mit anderen - sprich: medialen - Mitteln wahrgenommen werden, wie es sich in journalistischen und wissenschaftlichen Auseinandersetzungen nachvollziehen lässt (vgl. alJazeera English 2011; Lynch/Freelon/Aday 2014). Hingegen ist es mit der Vielzahl an unterschiedlichen ästhetisierenden Materialisierungen und Wahrnehmungen der Handy-Todesvideos in der Spätphase des Konflikts kaum mehr möglich, diese als eine ssanfte Waffe im Sinn der Abwesenheit einer Anwendung von Gewalt zu sehen. Vor allem dann nicht, wenn sich in dieser Wahrnehmung die genuinen Dokumente einer um ihr Leben videografierenden syrischen Zivilbevölkerung mit mokierenden, schockierenden, abstoßenden und horrorhaften Bildern des Todes ver- 
flechten. Die ein- und ausschließenden, sichtbar und unsichtbar machenden, betrachtbar und unbetrachtbar machenden ästhetischen und diskursiven Praktiken einer politischen Macht und der seiner Gegenspieler treten hierbei als Regulierungen des kippenden Moments auf. Sie sind Versuche der Verhinderung oder Steuerung des Umschlagens in eine bestimmte Wahrnehmung und Bedeutung eines einzelnen oder einer Menge von Handy-Todesvideos. Diese Ein- und Ausschlüsse lassen sich in den Erzählungen nachvollziehen, die mit den Handy-Todesvideos hervorgebracht werden, auf diese rekurrieren und sie in der einen oder anderen Weise bedeuten.

Die Narrative des Syrienkonflikts innerhalb des Phänomens der HandyTodesvideos lassen sich hierbei unter drei Zeitmodi fassen, die erzählen, wie der Syrienkonflikt im Handy-Todesvideo gestern wahrgenommen wurde, heute wahrgenommen wird und morgen wahrgenommen werden könnte. Anders als es diese dreischrittige Temporalität des Syrienkonflikts im Handy-Todesvideos zunächst suggeriert, ist das Verhältnis dieser Narrative zueinander weder im Sinn einer Abfolge noch einer vollständigen Ablöse zu sehen, sondern als eine überlagernde Gleichzeitigkeit. Das Gestern schwingt im Heute nach, ebenso wie Gestern und Heute das Morgen in Schwingung versetzen und das Morgen in den Hoffnungen und Versprechungen von heute und gestern bereits mitschwingt und mitschwang. Die abschließende Zusammenführung der sichtbargewordenen Narrative des Syrienkonflikts über eine solche temporale Kartierung ist jedoch insofern treffend, als dass sie die Begegnung und Wahrnehmung von mir als wissenschaftliche Betrachterin mit den Handy-Todesvideos nachvollzieht, die sich von den Anfängen des Syrienkonflikts im Frühjahr 2011 über heute Anfang/Mitte 2019 und bis zum unbestimmten morgen erstreckt.

Gestern. Im Handy-Todesvideo setzte der Syrienkonflikt als ein widerständiger Überlebenskampf ein. Eine syrische Zivilbevölkerung stellte sich mit den Bildern ihres Sterbens einer elliptischen Bildpraxis des syrischen Regimes entgegen: aufgenommen mit einfachen Handykameras, verbreitet über das Internet und gerichtet an uns als Angehörige einer gemeinsamen Opfer-, Täter- und Zeugenschaft von Filmenden, Aufnahme/Kamera und Betrachtenden. Mit ihrer eliptischen Bildpraxis zeichnete das Regime zunächst mitunter erfolgreich, wie es in den Gesprächen mit Syrern nacherzählt wurde, ihr Bild eines abwesenden Konflikts und einer kontrollierten, wenn auch von äußeren Kräften bedrohten Situation in Syrien. Keine Straßenkämpfe, keine Unterdrückung, keine innere Zerrüttung, keine Gewalt, keine Revolution waren in ihren Bildern zu sehen. Dem stellten die syrischen Videografierenden ihr eigenes Bild des Konflikts gegenüber: ein Selbstporträt `Syrien«. Über die Handy-Todesvideos schrieben sie sich nicht nur in den Syrienkonflikt als Antagonisten der politischen und unverhältnismäßigen Gewalt des syrischen Regimes ein. Sie schrieben sich auch in eine Zukünftigkeit des Konflikts ein, in dem sie sich mit ihrer widerständigen Videografie der Verschiebung in das Außerhalb eines po- 
litischen und rechtlichen Raumes widersetzten. Im Sinn einer vorwegnehmenden Zeitlichkeit riefen sie eine mögliche Gerichtbarkeit und Geschichtlichkeit ihrer Tode mit den Handy-Todesvideos auf. Sie brachten das Bild eines asymmetrischen Konflikts hervor, in dem die Macht des Bildes und des Affekts einer politischen Macht gegenübertritt. In dieser Erzählung des Syrienkonflikts stellt sich eine Gewalt mit anderen Mitteln einer militärischen Gewalt gegenüber und bricht das Bild des syrischen Regimes aus seinem feinjustierten Rahmen. Diese alternative Form der Gewalt manifestiert sich als ein ästhetisch-imaginär wirksamer, gewaltvoller Kamerablick, der eine bestimmte Perspektive des Syrienkonflikts hervorbringt und an ein Wir von Betrachtenden richtet. Das Aufbrechen der restriktiven Kadrierung des syrischen Regimes tritt dabei als ein Effekt der Sichtbarwerdung eines alternativen Narrativs des Syrienkonflikts in Erscheinung. Es ist eine Geschichte des Überlebens und Sterbens, die gemeinsam von den syrischen Videografierenden, der Aufnahme/Kamera und den Betrachtenden erzählt wird. In der digitalen Onlineumgebung mit seiner Ubiquität an Videos, die sich in immer verändernden Weisen zur Schuss- und Gegenschusseinstellung zusammenfügen, ist dieses Narrativ stets offen für alternative Erzählstränge.

Mit Verschärfung des Konflikts und dem Auftritt immer weiterer Akteure, die eine eigene videografische Praxis der gegnerischen Diffamierung und Diskreditierung und der eigenen Überhöhung und Akkreditierung etablierten, wurde dieses alternative Narrativ zunehmend mit den Geschichten eines wechselseitigen Vernichtungskampfes überlagert. Zudem zeigte sich ein narratives Eigenleben der Handy-Todesvideos, das außerhalb der Ein- und Ausschlussmöglichkeiten einzelner Akteure steht und sich auch gegen das Selbstporträt der widerständig videografierenden Syrer richtet. Die Wahrnehmung eines solchen Fremdporträtierens ließ den Syrienkonflikt im Handy-Todesvideo zunehmend als einen ausweglosen anomischen Bürgerkrieg sichtbar werden, in dem Horror zur Realität wird. Diese horrorhafte Realität wird in diesem Narrativ mit den Handyvideos selbst vorangetrieben, wenn diese im Sinn von Doppelagentinnen sowohl als Anzeigende und Verurteilende als auch als Mitbegründende des Horrors von Menschenrechtsund Tabuverletzungen, von identitären und körperlichen Verletzungen auftreten. Die Hoffnungen, Wünsche und Versprechungen, die mit den Handy-Todesvideos an ein zukünftiges Syrien gerichtet wurden und werden, überlagerten sich so mit Enttäuschung, Verzweiflung und Entmutigung, wie es in drei der Gespräche mit Syrern erzählt wurde. Diese wurden mit Ausbruchversuchen aus dem restriktiven Rahmen dieses Fremdporträts `Syrien • beantwortet, die nicht nur die Expositionsverweigerung gegenüber den Videos, sondern in letzter Instanz auch das Verlassen von (oder gar die Flucht aus) Syrien beinhalten.

Heute. Das Selbstporträt >Syrien ist heute nicht verschwunden. Weder ist es von der Bildfläche verschwunden, denn die Handy-Todesvideos von damals existieren weiter im Netz und erscheinen immer wieder neben aktuellen Videos auf 
der Bildschirmoberfläche - wann immer es der Vorschlagsalgorithmus einer Videoplattform in Antwort auf eine Suchanfrage und/oder einen Wiedergabeverlauf (engl.: ’watch history`) zulässt. Noch ist es aus der Wahrnehmung der widerständigen Syrer verschwunden. Ihre enttäuschten Wünsche und Hoffnungen, die sie an den Widerstand und die Handyvideos richteten, schwingen zusammen mit nicht erfüllten Versprechungen, die durch die Handyvideos an sie gerichtet wurden, in ihrer Wahrnehmung des Syrienkonflikts weiterhin mit. Ebenso bedeuten sie auch unsere Wahrnehmung des Konflikts mit (wenn auch vielleicht mit schwindender Vehemenz). Doch es rücken immer stärker Videos und Wahrnehmungen in den Vordergrund, die sich der Unterscheidung von politischen und ästhetisierenden Motivationen, von Realität und Fiktion widersetzen bzw. diese stören und eine Virtualität des Todes, des Syrienkonflikts und des Handyvideos aufrufen. Diese aufgerufene Virtualität beinhalten dabei auch ein Moment des Abjekten und Obszönen: der Tod als Endzustand und als Auslöser des Neustarts; der Syrienkonflikt als Bürgerkriegsschauplatz und als Virtual-Reality-Schlachtfeld; das Handyvideo als originales Rohmaterial und als fabriziertes Videowerk. Die gemeinsame Opfer-, Täter- und Zeugenschaft von Filmenden, Aufnahme/Kamera und Betrachtenden schlägt zu einem Kollektiv an betrachtenden Mit-Todesleidenden, Mit-zuVerantwortenden und Mit-Tätern um.

Überlagert wird das Selbstporträt eines widerständigen Überlebenskampfes auch von einer Blickverschiebung, die im Gestern ansetzte und bis heute anhält: Der Auftritt des IS als neuer Protagonist einer horrorhaften Konfliktgeschichte in und um Syrien lässt nicht mehr eine syrische Zivilbevölkerung im Fokus einer ästhetischen, diskursiven und existentiellen Gewaltanwendung stehen. Er holt vor allem den Westen ins Zentrum einer lebensbedrohenden Adressierung in und mit den Handy-Todesvideos. Das Wir einer gewalterfahrenden und -anwendenden Gemeinschaft von Filmenden, Aufnahme/Kamera und Betrachtenden wird auf eine lebensbedrohende Verbindung von Gefilmten, Adressierten und Betrachtenden verschoben. Die Geste der Gewalterfahrung und -anwendung richtet sich dabei nicht bloß von außen an dieses Wir. In einer geradezu narzisstischen Umkehrung im Rahmen rechtspopulistischer Bewegungen kippt sie in diesem Narrativ zu einer nach innen gerichteten Gewalterfahrung.

Neben der Wahrnehmung eines fremdporträtierten Syrienkonflikts mit den Handy-Todesvideos lebt das Selbstporträt >Syrien spielen fort. Hier zeichnet eine syrische Widerstands- und Flüchtlingsbewegung nicht nur >ihr Bild des Syrienkonflikts. Sie trägt die politischen Ansprüche eines einst in der Öffentlichkeit der Straße, des Internets, der Kunst und des Kinos geführten Überlebenskampfes in die Öffentlichkeit einer Gaming-Community. Auch andere Konfliktakteure bedienen sich dieser Form der Geschichtserzählung und -schreibung, um ihre Perspektive auf den Syrienkonflikt und ihren Interessen in diesem eine weitere Form der öffentlichen Sichtbarkeit zu verschaffen. Dies erfolgt 
bspw. im Fall von russischen Affiliationen von der hohen Warte auf ein steril distanziertes Kriegsstrategiespielfeld aus oder im Fall von libanesischen Affiliationen von der Unmittelbarkeit eines Face-to-Face-Straßenkampfes aus. In diesen Videospieladaptionen materialisiert sich die Ununterscheidbarkeit einer Ästhetisierung und Politisierung des Todes wie auch einer Realität und Fiktion des Syrienkonflikts.

Morgen? Erwartet uns eine anhaltende Virtualisierung des Syrienkonflikts und des Todes, wenn der Syrienkonflikt nicht mehr nur innerhalb einer Politik der Bedeutung der Handy-Todesvideos, sondern verstärkt auch innerhalb einer Politik der Bedeutung von Videospielen ausgetragen wird? Werden unterschiedliche Konfliktakteure ihre politischen Ansprüche weniger auf der Straße und mit Videos in sozialen Medien performieren, weniger über filmische Adaptionen in eine Öffentlichkeit des Kinos, von Festivals und Kunsträumen transportieren, als in und mit einer Virtual Reality? Wer wird dann das adressierte Wir ihrer politischen Ansprüche, der Verletzung und Verwerfung sein? Die in Syrien verbleibenden Syrer? Die geflüchteten Syrer in Europa? Die westlichen Bürger unterschiedlicher politischer und nationaler Spektren? Wie wird das Verhältnis eines Fremdporträtierens und Selbstporträtierens wahrgenommen werden? Werden die Syrer eine Betrauerbarkeit, Gerichtbarkeit und Geschichtlichkeit ihrer Leben und Tode - >ihres` Syriens herstellen und aufrechterhalten können? Oder wird diese von der Betrauerbarkeit, Gerichtbarkeit und Geschichtlichkeit der Leben und Tode anderer, direkter oder indirekter Konfliktbeteiligter und -betroffener - eines sanderen`Syriens - überlagert und ausgelöscht werden?

\section{2 ,Diffraction revisited}

Unter einem diffraktiven Leitbild werden die Narrative und Figurationen sichtbar, die, in Haraways (1997: 64) Worten, uns bewohnen und die wir bewohnen. Sie konstituieren das Wir innerhalb dessen Kommunikation möglich wird. Es ist ein performativ hergestelltes und nicht festgeschriebenes Wir, das sich, wie wir in dieser Arbeit gesehen haben, innerhalb eines figurativ-narrativen Zusammenspiels ästhetischer und diskursiver Variablen verschieben kann. Die Figurationen wie auch die Narrative haben dabei eine rahmende Funktion: Einerseits sind sie Ermöglichungen des Sichtbaren. Andererseits sind sie Begrenzungen des Sichtbaren. In einer diffraktiven Forschungsperspektive sind Narrative und Figurationen gemeinsam starke analytische Werkzeuge, die die Kartierung der von den betrachteten Ereignissen ausgehenden Diffraktionsmuster und Differenzeffekte möglich machen. Bestimmte Ereignisse rücken damit überhaupt erst in den Bereich des Sichtbaren - sprich: in den Blick der diffraktiven Forschungsperspektive. Die in den einzelnen Kapiteln kartierten Diffraktionsmuster - ausgehend von je spezifischen Ereignissen an Videos, Nachrichtensendungen und -artikeln, Kunstper- 
formances, Dokumentar- und Experimentalfilmen - stehen gleichzeitig innerhalb und außerhalb des Phänomens der Handy-Todesvideos. Sie bringen das Phänomen in unserem beugenden Blick einer diffraktiven Betrachtung hervor. Gleichzeitig treten sie erst aus unserer Betrachtung heraus als eng verflochtene, aufeinander verweisende und einander bedeutende Muster von Ereignissen, Figurationen und Narrativen in Erscheinung.

Wie jede wissenschaftliche Perspektive ist die diffraktive Forschungsperspektive dabei zugleich ein- und ausschließend. Sie produziert Sichtbarkeiten wie auch Unsichtbarkeiten. Sie stellt Betrachtbarkeiten her wie auch Unbetrachtbarkeiten. Un-/Sichtbarkeiten und Un-/Betrachtbarkeiten entstehen in der diffraktiven Forschungsperspektive auf das Phänomen der Handy-Todesvideos vor allem aus der Situiertheit der wissenschaftlichen Betrachtung und des Phänomens selbst heraus. So zeigt die diffraktive Betrachtung in dieser Arbeit einerseits meinen Blick als westliche Akademikerin, die keine direkten Affiliationen oder Verbindungen zur syrischen Kultur und Sprache hat. Das Phänomen der Handy-Todesvideos nehme ich vor allem durch mich selbst wahr: durch meine eigene soziale, kulturelle, sprachliche und wissenschaftliche Markierung, die eine eigene Ordnung der Sichtbarkeit und Betrachtbarkeit aufruft. Andererseits ist das Phänomen innerhalb einer medialen Onlineumgebung situiert, deren regulierenden Algorithmen bereits Un-/Sichtbarkeiten und Un-/Betrachtbarkeiten hervorbringen. So gehen schon mit einer anfragenden IP-Adresse und der Eingabesprache auf Videoplattformen und Suchmaschinen bestimmte Ein- und Ausschlüsse einher. Aber auch die den Videos allgemein anhaftende Flüchtigkeit, die auf der inhärenten Unbeständigkeit und Dispersität einer digitalen Onlineumgebung beruht, bedingen Ein- und Ausschlüsse von Ereignissen in der Betrachtung. Denn die Linkadressen, die in einem Moment noch zu einem Video führt, kann im nächsten schon ins Leere laufen, weil Videos oder Accounts gelöscht oder gesperrt wurden, weil Videos `umziehen zu einer anderen Adresse oder einem anderen Account mit anderen Affiliationen, weil die Videos innerhalb der Funktionen einer Plattform in einen Wiedergabe- und Vorschlagsfluss eingebunden sind, der so schnell wie er uns zu einem Video getragen hat, uns auch schon zum nächsten Video fortträgt.

Zur selben Zeit stellt die soziopolitische Verortung des Phänomens Un-/Sichtbarkeiten und Un-/Betrachtbarkeiten her. Im Verlauf des Syrienkonflikts haben sich seine soziopolitischen Parameter mit dem Auftreten neuer und dem Verschwinden alter Konfliktakteure sowie mit der in 2015 extremen Fluchtbewegung von Syrien und Irak nach Europa (vgl. Flack/Hanewinkel/Latz 2017) signifikant verschoben. Vor allem die soziopolitische Verortung der wissenschaftlichen Betrachtung spielt hier hinein, die aus einem bestimmten soziopolitischen Umfeld - hier: der Bundesrepublik Deutschland - heraus erfolgt und mit der syrischen Fluchtbewegung nach Europa an Bedeutungsschwere gewonnen hat. Der soziopolitische, soziokulturelle, wissenschaftliche und technologische Standort, von 
dem aus die Betrachtung erfolgt, ist in den kartierten Diffraktionsmustern dieser Arbeit nicht wegzudenken. Ändert sich dieser, ändert sich das Phänomen der Handy-Todesvideos.

Die inhärente Unabgeschlossenheit der hier kartierten Diffraktionsmuster macht diese Arbeit dabei anschlussfähig für Betrachtungen weiterer Ereignisse unterschiedlicher Materialisierungen, die das Phänomen nachträglich in einer anderen Weise bedeuten. Diese Ereignisse können eigene Diffraktionsmuster zeichnen, sich mit den hier gezeichneten und noch zu zeichnenden Diffraktionsmustern überlagern und die figurativen Verflechtungen innerhalb des narrativen Netzes des Phänomens der Handy-Todesvideos neuordnen und erweitern. Damit ist diese Arbeit auch offen für alternative Erzählungen und Wahrnehmungen des Todes, des Syrienkonflikts und des Handyvideos im Phänomen der HandyTodesvideo. In einer eigenen und bedeutenden Weise hat sich diese narrative Offenheit bereits in den Artikulationen direkt und indirekt konfliktbetroffener Syrer in dieser Arbeit materialisiert. Eine Ausweitung der darin verfolgten ethnografischen Perspektive unter Zugriff auf medienanthropologische Ansätze, die sich auf die Veränderung der gelebten und wahrgenommenen Realität von Konflikten mit und durch Medien fokussieren (vgl. Budka/Bräuchler 2020; Meis 2020), verspricht unseren beugenden Blick für solche alternative Erzählungen und Wahrnehmungen weiter zu öffnen.

Dieselbe inhärente Unabgeschlossenheit der Diffraktionsmuster bedingt jedoch auch, dass das Phänomen der Handy-Todesvideos sich im Moment seiner Kartierung bereits verändert hat und weiter verändern wird. Gegenüber diesem ständigen In-Bewegung-seins kann der Anspruch und die Aufgabe einer diffraktiven Betrachtung nur sein zu zeigen, wie unterschiedliche, ineinandergreifende, aber auch gegeneinanderstehende Narrative und Figurationen sich im Moment der Betrachtung zu Diffraktionsmustern verbinden und welche Politiken der Bedeutung damit sichtbar werden. Mein Anspruch ist daher gerade nicht der einer bescheidenen oder zurückhaltenden Betrachterin - eines unsichtbaren und geschichtsfreien »modest witness (Haraway 1997: 24), wie ihn Haraway in der modernen Wissenschaftsschreibung einer »culture of no culture« (ebd: 23) zurückweist. Sie ist der einer markierten und markierenden Betrachterin, die den figurativen Erzähl- und Lesbarkeiten - den »imaginary configurations« (ebd.: 2) - der HandyTodesvideos an- und nachhängt. Dieser Anspruch ist herausfordernd und riskant: Er ist herausfordernd, weil damit ein kritisches Moment in die Betrachtung eingeschrieben ist, die diese auch selbst einschließt. Er ist riskant, weil damit nicht nur die Produktion von Un-/Sichtbarkeiten und Un-/Betrachtbarkeiten im Rahmen einer figurativ-narrativen Praxis der Wissens- und Bedeutungsgenerierung kartiert wird. Er ruft auch eigene Un-/Sichtbarkeiten und Un-/Betrachtbarkeiten hervor.

Diese eigenen Un-/Sichtbarkeiten und Un-/Betrachbarkeiten $\mathrm{zu}$ begehen und herauszufordern, ist der besondere Beitrag einer diffraktiven Perspektive. Als wis- 
senschaftliche Forschungsperspektive ist Diffraktion eine zugleich narrative, bildliche und politische Technologie, um folgenreiche Geschichten und Bedeutungen zu produzieren. Als rahmende Metapher steht Diffraktion für eine andere Art des kritischen Bewusstseins, das sich nicht der Wiederholung des Gleichen verschreibt, sondern einen Unterschied machen möchte. (vgl. ebd.: 273) Dieses Leitbild und mit ihm die Forschungsperspektive dieser Arbeit wollen entsprechend herausfordernd gegenüber etablierten und weniger etablierten Sichtweisen auf den Tod, den Syrienkonflikt und das Handyvideo sein und sich dabei selbst dem Risiko aussetzen, herausgefordert zu werden. Das diffraktive Leitbild überträgt und pointiert dabei selbst Bedeutung und ist eingebunden in eine diffraktive Pragmatik der kontextabhängigen Bedeutungsgenerierung - eines "meaning-in-the-making« (ebd.: 126), in dem sich die diffraktive Betrachtung einschreibt. Entsprechend macht das In-Frage-stellen des diffraktiven Leitbildes und der sich daraus ableitenden Forschungsperspektive dieser Arbeit gerade auch sichtbar, wie es bereits Judith Butler (2009: 10) für eine begrenzende und zugleich ermöglichende Rahmung formuliert hat, dass das damit beschriebene Phänomen niemals vollständig in diesem Bild enthalten sein kann. Schon immer steht etwas außerhalb des Bildes, das sein Innerhalb möglich und wahrnehmbar macht. Daher ist es notwendig, dieses Leitbild immer auch zu verlassen, um den Blick für andere Sichtweisen, andere Ereignisse und andere Phänomene zu öffnen.

Die nachverfolgten figurativen Verflechtungen verweisen die diffraktive Betrachtung dabei bereits auf ein Außerhalb ihrer Perspektive. Dort finden sich Schnittstellen und Affinitäten, die alternative und ergänzende Zugänge zum Phänomen der Handy-Todesvideos bereitstellen können. Ebenso können diese von einem diffraktiven Zugang, wie er diese Arbeit geleitet hat, profitieren. So sprechen die Figurationen des Todesschocks, des Schusses und des Todeshorrors Zugänge filmwissenschaftlicher Fokussierungen an, um die ästhetischen Aspekte der Handy-Todesvideos, die sich dem Film entlehnen und sich in den Handyvideos in einer eigenen Weise zusammenfügen, zu adressieren. In dieser Arbeit sind der extrinsische Gegenschuss und die elliptischen Todesinszenierung als ästhetische Mittel am Schnittpunkt von Film und Video sichtbar geworden, die sich mit einer filmwissenschaftlichen Ausrichtung eingehender betrachten und in Hinblick auf Fragen der Medienkonvergenz begehen ließen. Ebenso bieten die Überführungen von Handyvideos in filmische Formate, wie es EAU ARGENTÉE leistet, aber auch darüberhinaus in anderen filmischen Arbeiten zum Arabischen Frühling der Fall ist (z.B. The Uprising (2013) (B/UK, R: Snowdon)), Anschlusspunkte für weiterführende Fragestellungen an der Schnittstelle von Filmtheorie und Kulturwissenschaft. Insbesondere das Aufgreifen des in Bezug auf EAU ARGENTÉE herausgestellten kritischen Moments einer Überschreibung von Amateurvideoaufnahmen aus dem Internet mit einer cineastischen Intention, mit cineastischen Ästhetiken und Diskursen, kann hier gewinnbringend sein. 
Mit der Figuration der Waffen-/Kameragewalt finden sich u.a. Anschlusspunkte für mediengeschichtlich orientierte Ansätze, wenn die historisch gewachsene Verbundenheit von Waffe und Kamera und ihre spezifische Vereinigung in den Handy-Todesvideos zentriert werden. Vor allem die vollzogene Bewegung der Auffächerung und Wiederzusammenführung des Phänomens der Handy-Todesvideos in die Aspekte des Todes, des Handyvideos und des Syrienkonflikts ist hier ein vielsprechendes Vorgehen, um die apparative, ästhetische und diskursive Vereinigung von Handy und Waffe zu betrachten. Video-Martyrium und Todesleid sprechen vor allem ikonografisch und ikonologisch ausgerichtete Forschungen an, als dass sie einerseits auf den Prozess der Ikonisierung und andererseits auf den symbolischen Gehalt der Handy-Todesvideos verweisen. Hervorzuheben sind hierfür die in Erscheinung getretenen ästhetischen und diskursiven Verschiebungen von einem auf den Tod verweisenden Märtyrervideo zu einem auf das Überleben gerichteten Video-Martyrium. Ebenso ist hier die Verschiebung von dem verbildlichten Fokus eines stattfindenden oder stattgefundenen Leidens des Sterbenden oder Toten auf das Leiden der beistehenden Gefilmten und Filmenden anzumerken. Letztere deutete sich prominent in der Betrachtung von EAU ARGENTÉE und dem Modus einer erzählerischen Mitsicht an und bietet vielsprechende Anknüpfungspunkte.

Innerhalb der Figuration der Zeugenschaft zeigen sich vor allem Anschlüsse für den interdisziplinären Bereich der >Memory and Testimony Studies`, wenn dort bspw. Fragen sich verändernder Zeugenschaftskonstellationen und -szenarien oder der Refiguration eines kulturellen oder kollektiven Gedächtnisses im Mittelpunkt stehen. Mit den Handy-Todesvideos zeigt sich hier ein neuer Modus des Überlebenszeugnisses: das widerständige Nicht-Überlebenszeugnis zwischen Filmenden, Handykamera bzw. -aufnahme und Betrachtenden, das sich als standhafter digitaler Bildkörper dem Auslöschungswillen einer politischen Macht entgegenstellt. Diese alternative Form des Zeugenschaftsszenarios verspricht neue Impulse für Forschungen im Bereich der >Memory and Testimony Studies $\prec$ Das Videoerlebnis TOD schafft mit seinen Verweisen zur virtuellen und spielerischen Wahrnehmung des Todes im Syrienkonflikt vor allem Anschlussstellen für eine medienkulturwissenschaftlich ausgerichtete `Game Studies $<$. Hier verspricht z.B. das $>$ Project Syria< im Rahmen des >Immersive-journalism<-Konzepts (vgl. de la Peña et al. 2010, 2014) produktive Schnittstellen. Gemeinsam mit der Figuration der Lebensbedrohung bieten sich in Hinblick auf die in den Kommentaren zu YouTubeVideos aufgerufenen ästhetischen und politischen Motivationen Anschlüsse für sozialwissenschaftliche Forschungen insb. im Feld der Sozialpsychologie. Auch lassen sich für Forschungen im Bereich der sozialen Bewegungen weitere Auseinandersetzungen hinsichtlich Fragen nach einer sich verändernden Protestperformance, -öffentlichkeit und -teilhabe vorstellen, wie sie sich bspw. bei Cecile Boëx (2016) andeuten. 
Die in den Handy-Todesvideos stets mitschwingenden Affekte und deren Regulierungen im Rahmen einer Politik der Bedeutung lassen insbesondere an Forschungen im Bereich einer >Feminist Cultural Studies of Emotion and Affect $<$ denken, wie sie Sara Ahmed in The Cultural Politics of Emotion (2014 [2004]) verfolgt. Sieht Ahmed Emotionen hier als unsere Antwort gegenüber Objekten und Subjekten als eine Angelegenheit des Kontakts mit anderen - die Oberflächen und Grenzen hervorbringt (vgl. ebd.: 10), können die hier kartierten Erzählungen und Bedeutungen in einer Weise adressiert werden, die feministische Lesarten mit soziopolitischen und soziokulturellen Ansätzen und medienwissenschaftlichen Fokussierungen zusammenbringt. Hiermit ließ sich bspw. die Frage danach betrachten, inwiefern sich Affektivitäten als medientechnologisch bedingte Materialisierungen von politischen Auseinandersetzungen um die Authentizität von Todesvideos beschreiben lassen, wie es sich im Zusammenhang der Enthauptungsvideos in dieser Arbeit formulieren ließ. Ahmed entwickelt zudem die Vorstellung einer Narrativität von Emotionen als die Konvertierung von Emotionen unter bestimmten Lesarten und die ursächliche Zuschreibung von Emotionen an Andere (vgl. ebd.: 13). Diese Vorstellung kann Affektanrufe und eine Politik des Affekts als »histories of articulation« (ebd.: 2) nachvollziehbar machen. In dieser Arbeit zeigen sich diese Affektanrufe und die damit verbundenen Politiken vor allem in dem widerständigen Überlebenskampf einer videografierenden syrischen Zivilbevölkerung, in den Imaginationen einer kollektiven Opfer-, Täter- und Zeugenschaft von Filmenden, Aufnahme/Kamera und Betrachtenden, und in den Inszenierungen jihadistischer und rechtspopulistischer Identitätskämpfe als solidarisierende, überzeugende oder einschwörende Erzählstränge. Die Verknüpfung einer diffraktiven Forschungsperspektive mit einer feministischen Betrachtung von Emotionen und Affekten verspricht sowohl den anziehenden als auch den abstoßenden Effekt dieser Narrative sichtbar zu machen und dabei die Produktion der eigenen Un-/Sichtbarkeiten und Un-/Betrachtbarkeiten nicht aus dem Blick zu verlieren. 
